\title{
Error rate degradation due to switch crosstalk in large modular switched optical networks
}

Saxtoft, Christian; Chidgey, P.

Published in:

I E E E Photonics Technology Letters

Link to article, DOI:

10.1109/68.229821

Publication date:

1993

Document Version

Publisher's PDF, also known as Version of record

Link back to DTU Orbit

Citation (APA):

Saxtoft, C., \& Chidgey, P. (1993). Error rate degradation due to switch crosstalk in large modular switched optical networks. I E E E Photonics Technology Letters, 5(7), 828-831. https://doi.org/10.1109/68.229821

\section{General rights}

Copyright and moral rights for the publications made accessible in the public portal are retained by the authors and/or other copyright owners and it is a condition of accessing publications that users recognise and abide by the legal requirements associated with these rights.

- Users may download and print one copy of any publication from the public portal for the purpose of private study or research.

- You may not further distribute the material or use it for any profit-making activity or commercial gain

- You may freely distribute the URL identifying the publication in the public portal

If you believe that this document breaches copyright please contact us providing details, and we will remove access to the work immediately and investigate your claim 


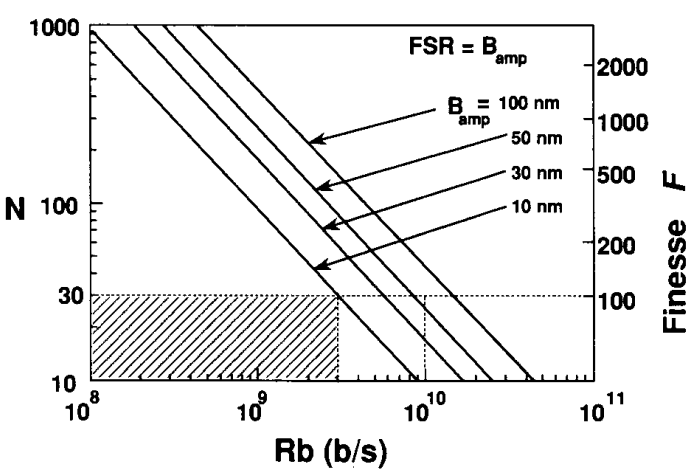

Fig. 5. The characteristics of the maximum number of channel as a function of the $F$.

The characteristics of the maximum number of nodes $N$ (which coincides with the number of frequencies) as functions of $R_{b}$ and $F$ are shown in Fig. 5. For example, for $F$ is 100 , the limitation of $N$ is 30 for the $R_{b}$ of less than $3 \mathrm{~Gb} / \mathrm{s}$. If more than 30 frequencies are required to the proposed network, finesse of FFP filters must be increased more than 100 . On the other hand, $B_{\text {amp }}$ of more than $50 \mathrm{~nm}$ is required for the $R_{b}$ of higher than 10 $\mathrm{Gb} / \mathrm{s}$.

\section{CONCLUSION}

A logical star ring network based on OFDM-ADM employing a FFP filter and an optical circulator is proposed. Focusing on the crosstalk, the maximum number of nodes for the $1 \times N$ ring network was estimated concerning with the finesse of the FFP filter and the bandwidth of optical amplifiers. The criterion of total crosstalk was estimated to be $-11.7 \mathrm{~dB}$ based on $100 \mathrm{GHz}$ spaced, 622 $\mathrm{Mb} / \mathrm{s}$, three-channel IM-DD transmission experiment. As a result, the limitation of $N$ was estimated to be 30 for the $R_{b}$ of less than $3 \mathrm{~Gb} / \mathrm{s}$, which is mainly determined by the finesse of 100 .

\section{ACKNOWLEDGMENT}

The authors wish to thank Dr. Nosu and Dr. H. Ishio for their continuous encouragement.

\section{REFERENCES}

[1] M. I. Irshid et al., "A fully transparent fiber-optic ring architecture for WDM networks," J. Lightwave Technol, vol. LT-10, pp. 101-108, Jan. 1992.

[2] W. I. Way et al., "Optical power limiting amplifier and its application in an SONET self-healing ring network," J. Lightwave Technol., vol. 10, pp. 206-214, Feb. 1992.

[3] - "A self-routing WDM high-capacity SONET ring network," IEEE Photon. Technol. Lett., vol. 4, pp. 402-405, Apr. 1992.

[4] D. A. Smith et al., "Integrated-optic acoustically-tunable filters for WDM networks," J. Select. Areas Commun., vol. SAC-4, pp. $1151-1159,1986$.

[5] I. P. Kaminow et al., "FDMA-FSK star network with a tunable optical filter demultiplexer," $J$. Lightwave Technol., vol. 6, pp. 1406-1988, Sept. 1988.

[6] J. Stone et al., "Pigtailed high-finesse tunable fiber Fabry-Perot interferometers with large, medium and small free spectral ranges," Electron. Lett., vol. 23, no. 15, pp. 781-783, 1987.

[7] T. Matsumoto et al., "Polarization-independent optical circulator: An experiment," Appl. Opt., no. 19, pp. 108-112, 1980.

[8] H. Toba et al., "A conceptional design on optical frequency-division-multiplexing distribution systems with optical tunable filters," J. Select. Areas Commun., vol. SAC-4, pp. 1458-1460, 1986.

\title{
Error Rate Degradation Due to Switch Crosstalk in Large Modular Switched Optical Networks
}

\author{
Christian Saxtoft and Peter Chidgey
}

\begin{abstract}
A theoretical model of an optical network incorporating wavelength selective elements, amplifiers, couplers and switches is presented. The model is used to evaluate a large modular switch optical network that provides the capability of adapting easily to changes in network traffic requirements. The network dimensions are shown to be limited by the optical crosstalk in the switch matrices and by the polarization dependent loss in the optical components.
\end{abstract}

Manuscript received December 1, 1992; revised May 4, 1993.

C. Saxtoft is with the Center for Broadband Telecommunications, Electromagnetics Institute, Technical University of Denmark, 2800 Lyngby, Denmark.

P. Chidgey is with British Telecom Laboratories, Main Networks Division, Martlesham Heath, IP5 7RE Ipswich, England.

IEEE Log Number 9210438

\section{INTRODUCTION}

\footnotetext{
$\mathrm{T}$
} ELECOMMUNICATION networks of the future must be capable of adapting to rapid changes in the network traffic requirements. This is a consequence of the introduction of a number of new narrowband and broadband services with, at present, uncertain demands on bitrates, signal formats, etc.. Today, the development of telecommunication networks is constrained by the inflexible interface between the optical high-speed fiber interconnection networks, and the electronic terminals and switch nodes. The vast bandwidth potential of the optical fibre cannot be exploited easily since the existing elec- 
tronic interface is designed for specific multiplexing schemes and bitrates. Therefore, post installation changes will be expensive. Optical technologies may be employed to provide the required capacity and flexibility. Until now, advanced optical techniques for time switching and frequency switching are still immature compared to the electronic counterparts, whereas optical space switching and wavelength division multiplexing (WDM) [1] provide attractive solutions to some of the improved networking functions required.

\section{Optical Network ARchitecture}

A schematic architecture for an optical core network is shown in Fig. 1. The optical nodes are linked in a mesh configuration where transmission in opposite directions in the network is carried over two separate subnetworks. Optical isolators are assumed to eliminate problems caused by optical reflections. An optical path through the network will typically comprise a number of fiber transmission sections interconnected by optical network nodes incorporating optical space switches, optical amplifiers and WDM components. This network forms a high capacity optical transport layer of simple functionality with access to an electronic transport layer of limited bandwidth capable of providing a number of network management functions, drop-insert of new channels, etc.. The optical network is constructed in a modular fashion where each module consists of a network node and a length of fiber. If operation of the single network elements is independent of the overall network architecture, this approach allows simple overall network configuration and ease of upgrade and extensions.

In Fig. 2 a single network module (building block) is illustrated along with a schematic of an optical path through the network. An optical building block comprises wavelength selective elements for improved capacity and flexibility, amplifiers for signal level restoration, a splitter and a small switch for path protection, an optical crosspoint switch matrix, and a length of fiber for network node interconnection. The signals at the input and at the output of a network building block must be maintained at the same level.

\section{Optical NeTwORK MOdel}

The dimension of the network is limited by a number of effects such as optical crosstalk, amplifier spontaneous emission, laser saturation, fiber nonlinearities, reflections, jitter accumulation, and signal bandwidth narrowing caused by filter concatenation. To get an appreciation of the limits to the number of building blocks that can be cascaded, a statistical model for the bit-error-rate (BER) at the receiver has been developed. Only the degradation caused by amplifier noise, receiver noise and switch crosstalk is considered. Wavelength crosstalk caused by non-ideal filters or amplifier saturation is not taken into account.

The optical amplifiers contribute noise due to the spontaneous emission of photons in the laser cavity, and at the

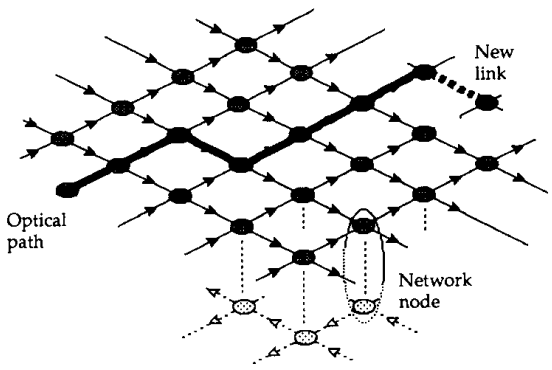

Fig. 1. Optical mesh network.

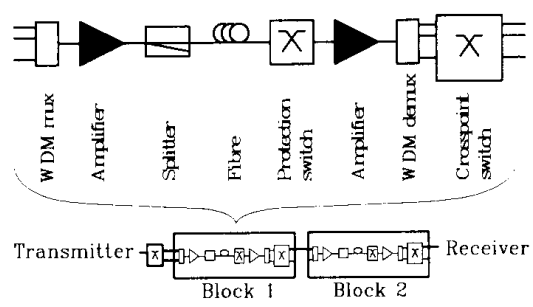

Fig. 2. Building blocks for optical mesh network.

network termination intrinsic receiver noise is added. Hence, the aggregate noise current variance can be expressed as

$$
\begin{aligned}
\sigma_{0 / 1}^{2}= & \sigma_{\text {signal }}^{2}+\sigma_{\text {dark }}^{2}+\sigma_{\text {spon }}^{2}+ \\
& \sigma_{\text {spon-spon }}^{2}+\sigma_{\text {signal-spon }}^{2}+ \\
& \sigma_{\text {thermal }}^{2}
\end{aligned}
$$

The model for the accumulated optical amplifier noise is based on [2] and [3]. The total optical crosstalk at the receiver is assumed to be dominated by the crosstalk of the optical switch elements in the network. For simplicity, these contributions, $I_{x i}$, are assumed to be equal. Hence, the maximum system crosstalk is given by

$$
I_{\text {crosstalk }}=I_{x 1}+I_{x 2}+I_{x 3}+\cdots \cdots+I_{x R}=R I_{x}
$$

where $R$ is the number of switch crosspoints in the optical path. This expression gives a worst case estimate of the aggregate optical crosstalk level at the receiver. In networks based on on /off modulated signals, the " 0 " state of the signal will not contribute to the crosstalk. If the crosstalk signals are assumed statistically independent, the number of " 1 "-state crosstalk signals is binomially distributed and takes on $R+1$ possible values with the probability $P_{B}\{r=i\}=R ! /\left(i !(R-i) ! 2^{R}\right)$ where $i=0,1$, $2 \cdots \cdots, R$.

The crosstalk is assumed to appear as background light at the receiver [4]. The BER may then be evaluated for each possible crosstalk level, $r I_{x}$, at the receiver. We get

$$
\begin{aligned}
\mathrm{BER}=\sum_{i=0}^{R} \frac{1}{2 \sqrt{2 \pi}}\left(\int_{Q_{0}}^{\infty} \exp \left(-y^{2} / 2\right) d y\right. \\
\\
\left.\quad+\int_{Q_{1}}^{\infty} \exp \left(-y^{2} / 2\right) d y\right) P_{B}\{r=i\}
\end{aligned}
$$


TABLE 1

Parameters Used IN Simulations

\begin{tabular}{|l|l|c|}
\hline Transmitter & Wavelength & $1.55 \mu \mathrm{m}$ \\
\hline \multirow{4}{*}{ PIN receiver } & Equivalent input noise & $3.2 \mathrm{pA} / \mathrm{Hz}$ \\
\cline { 2 - 3 } & Bandwidth & $1 \mathrm{GHz}$ \\
\cline { 2 - 3 } & Responsivity & $0.85 \mathrm{~A} / \mathrm{W}$ \\
\hline \multirow{4}{*}{ mux/demux } & Number of channels & Variable 1-32 \\
\cline { 2 - 3 } & Loss & $4 \mathrm{~dB}$ \\
\cline { 2 - 3 } & Bandwidth & Variable \\
\hline \multirow{3}{*}{$\begin{array}{l}\text { Protection } \\
\text { switch }\end{array}$} & Size & $2 \times 2$ \\
\cline { 2 - 3 } & Loss & $6 \mathrm{~dB}$ \\
\cline { 2 - 3 } & Crosstalk per crosspoint & $-20 \mathrm{~dB}$ \\
\hline \multirow{3}{*}{ srosspoint } & Size & $8 \times 8$ \\
\cline { 2 - 3 } & Loss & $10 \mathrm{~dB}$ \\
\cline { 2 - 3 } & Crosstalk per crosspoint & $-20 \mathrm{~dB}$ \\
\hline \multirow{3}{*}{ Amplifier } & Gain & $18 \mathrm{~dB}$ \\
\cline { 2 - 3 } & Aggregate input power & $-17 \mathrm{dBm}$ \\
\cline { 2 - 3 } & Spon. emission factor & 1.5 \\
\cline { 2 - 3 } & Coupling loss & $0 \mathrm{~dB}$ \\
\hline \multirow{3}{*}{ Fibre } & Loss & $9 \mathrm{~dB}$ \\
\cline { 2 - 3 } & Maximum power in fibre & $-2 \mathrm{dBm}$ \\
\hline Splitter & Loss & $3 \mathrm{~dB}$ \\
\hline
\end{tabular}

where

$$
Q_{0}=\frac{\left|D-\left(I_{0}+r I_{x}\right)\right|}{\sigma_{0}}, \quad Q_{1}=\frac{\left|D-\left(I_{1}+r I_{x}\right)\right|}{\sigma_{1}}
$$

in which $D$ is the decision level and $I_{0 / 1}$ is the photo current for the " 0 " and " 1 " states respectively. The states are assumed to have equal probability. $D$ is chosen to minimise the total BER using a numerical approach, since the optimum threshold is analytically intractable.

\section{Optical Network Simulations}

The modular optical network shown in Fig. 2 has been simulated using this model. The network parameters used are given in Table I. The network performance has been modelled for two different switch matrix configurations [5]. The Benes structure is characterised by poor overall crosstalk suppression but requires only $2 \log _{2} \mathrm{~N}-1$ crosspoint stages for a $N \times N$ switch. The active splitter/active combiner tree structure features good crosstalk performance using $2 \log _{2} N$ crosspoint stages, however, the insertion loss is slightly increased. The Benes architecture is rearangable nonblocking and the tree architecture is strictly nonblocking. The performance of the two different network configurations is compared in Fig. 3 and 4. The aggregate number of crosspoint stages, $R$ is found by adding the number of stages for the individual switches along the path, see Fig. 2. The BER is calculated from (3). Further, the statistical crosstalk model is compared to a simplified on-off model where the system crosstalk with equal probability takes on the values 0 and $R I_{x}$ only.

The significant difference in the number of cascaded network blocks, 37 and 16, respectively, for a system with 16 wavelengths, at a BER of $10^{-9}$, is caused by the higher crosstalk suppression of the tree switch architecture. For

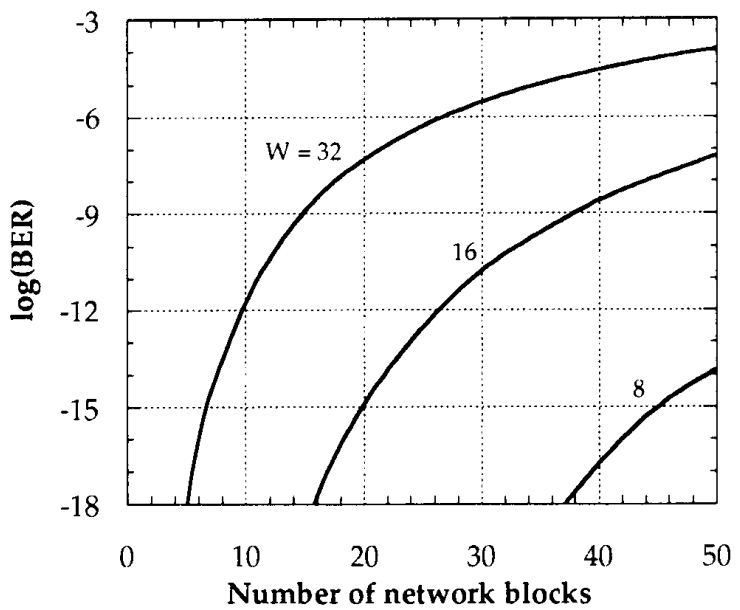

Fig. 3. Calculated BER for a modular optical network with active/active tree switch matrices. The number of wavelength channels, $W$, is 8 , 16 , or 32

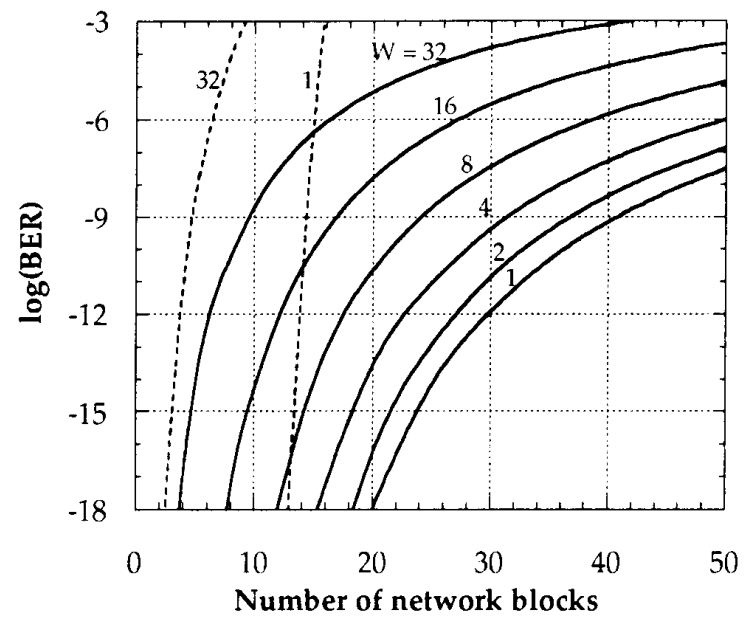

Fig. 4. Calculated BER for a modular optical network with Benes switch matrices. The number of wavelength channels, $W$, is $1,2,4,8,16$, or 32 . The bold line plots represent the statistical crosstalk model and the dashed line plots are results from the on/off crosstalk model.

this architecture the crosstalk will not affect the system performance even for a large number of concatenated networks block.

The insertion loss of the blocks is assumed to be adjusted to $0 \mathrm{~dB}$. However, the optical components may have an insertion loss depending on the polarisation state that cannot be compensated for. The influence of a $0.1 \mathrm{~dB}$ polarisation dependent loss for each switch crosspoint is illustrated in Fig. 5 and Fig. 6. This results in an overall insertion loss of $0.6 \mathrm{~dB}$ for network blocks based on the Benes switch matrix compared with $0.8 \mathrm{~dB}$ for the tree architecture based modules. For a system with 16 wavelength channels the maximum number of cascaded networks blocks is now 6-7 blocks for both switch architectures at a BER of $10^{-9}$. 


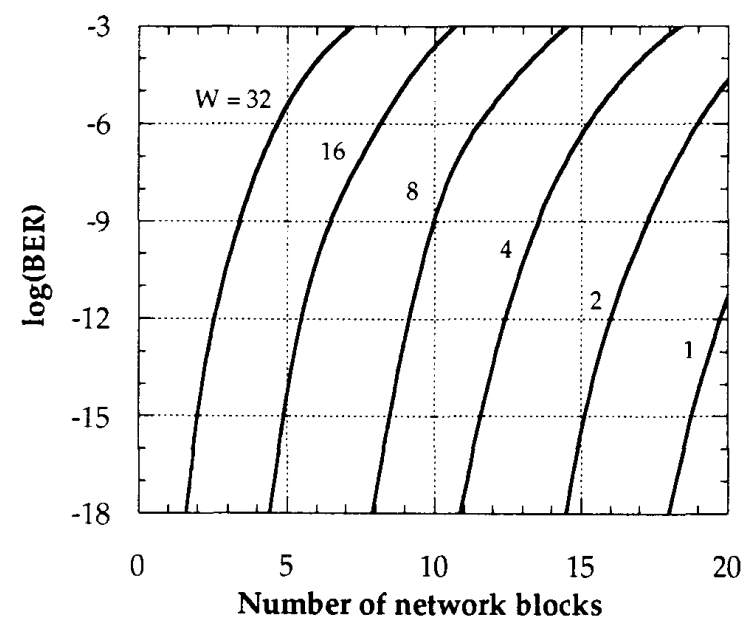

Fig. 5. Calculated BER for a modular optical network with active/active tree switch matrices. The number of wavelength channels, $W$, is 1,2 , $4,8,16$, or 32 . The influence of a $0.1 \mathrm{~dB}$ polarization sensitivity per switch crosspoint is included.

In large networks ( $R$ large) the level of the total crosstalk at the receiver is approximately Gaussian distributed with a variance of $\left(R I_{x}\right)^{2} / 4 R$. Hence, the signalto-noise-and-crosstalk-ratio can be defined as

$$
\frac{S}{N X} \equiv 20 \log \frac{I_{1}-I_{0}}{\frac{1}{2}\left(\sqrt{\sigma_{1}^{2}+\frac{\left(R I_{x}\right)^{2}}{4 R}}+\sqrt{\sigma_{0}^{2}+\frac{\left(R I_{x}\right)^{2}}{4 R}}\right)}
$$

Now, a $S / N X$ ratio of $21.6 \mathrm{~dB}$ corresponds to a BER of $10^{-9}$. Over the range $10^{-4}<\mathrm{BER}<10^{-12}$ the estimates of the BER derived from the $S / N X$ ratio approach are within $50 \%$ of the above numerical results obtained by the network model. This accuracy may in practice be sufficient to get an appreciation of the parameters limiting the network performance.

\section{CONCLUSIONS}

A switched optical network architecture based on optical modules linked in a mesh configuration has been presented and a model of this network has been devel-

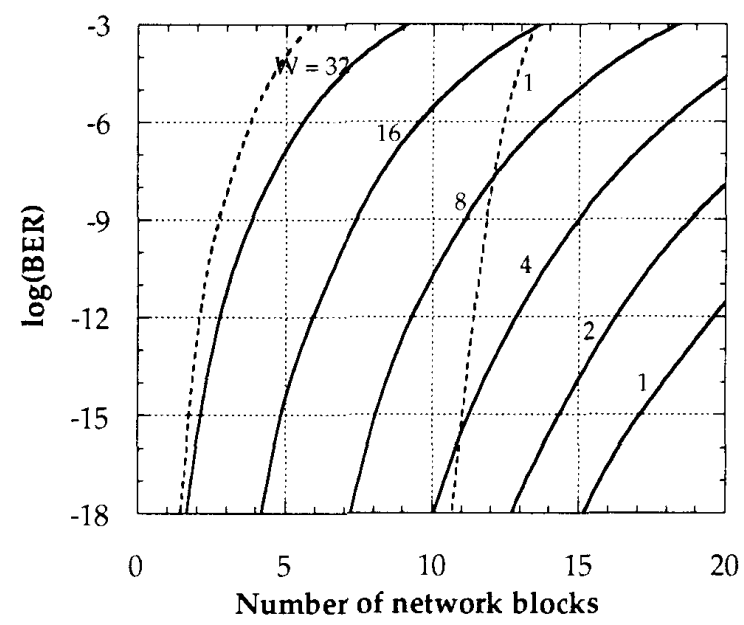

Fig. 6. Calculated BER for a modular optical network with Benes switch matrices. The number of wavelength channels, $W$, is $1,2,4,8,16$, or 32. The bold line plots represent the statistical crosstalk model and the dashed line plots are results from the on/off crosstalk model. The influence of a $0.1 \mathrm{~dB}$ polarization sensitivity per switch crosspoint is included.

oped. The importance of keeping the polarisation dependent insertion loss of the modules as low as possible is illustrated and the trade off between this requirement and the need for low crosstalk is analyzed. The modular networking approach with it's high capacity and increased flexibility makes it a promising solution for use in future transparent optical networks.

\section{REFERENCES}

[1] G. R. Hill, "A wavelength routing approach to optical communications networks," Brit. Telecom Technol. J., vol. 6, pp. 24-31, 1988.

[2] A. Lord and W. A. Stallard, "A laser amplified model for system optimization," Optical Quantum Electron., vol. 21, pp. 463-470, 1989.

[3] T. M. Mukai, Y. Yamamuto, and T. Kimura, " $S / N$ and error rate performance in A1GaAs semiconductor laser preamplifier and linear repeater systems," IEEE J. Quantum Electron., vol. 18, pp. 564-575, Oct. 1982

[4] P. Granestrand, L. Thylén, and G. Wicklund, "Analysis of switching employing a $4 \times 4$ switch matrix: Crosstalk requirements and system proposal," in Proc. ECOC'89, Gothenburg, 1989, pp. $276-279$.

[5] R. A. Spanke, "Architectures for guided-wave optical space switching systems," IEEE Com. Mag., vol. 25, no. 5, pp. 42-48, 1987. 